\title{
Estimação de componentes de co-variância para pesos corporais do nascimento aos 365 dias de idade de bovinos Guzerá empregando-se modelos de regressão aleatória ${ }^{1}$
}

\author{
Luciele Cristina Pelicioni², Lucia Galvão de Albuquerque ${ }^{2,3}$, Sandra Aidar de Queiroz ${ }^{2,3}$ \\ 1 Suporte financeiro da FAPESP. \\ 2 Departamento de Zootecnia - FCAV/UNESP - Jaboticabal, SP. \\ ${ }^{3}$ Bolsista CNPq.
}

RESUMO - Um total de 19.770 pesos corporais de bovinos Guzerá, do nascimento aos 365 dias de idade, pertencentes ao banco de dados da Associação Brasileira dos Criadores de Zebu (ABCZ) foi analisado com os objetivos de comparar diferentes estruturas de variâncias residuais, considerando 1, 18, 28 e 53 classes residuais e funções de variância de ordens quadrática a quíntica; e estimar funções de co-variância de diferentes ordens para os efeitos genético aditivo direto, genético materno, de ambiente permanente de animal e de mãe e parâmetros genéticos para os pesos corporais usando modelos de regressão aleatória. Os efeitos aleatórios foram modelados por regressões polinomiais em escala de Legendre com ordens variando de linear a quártica. Os modelos foram comparados pelo teste de razão de verossimilhança e pelos critérios de Informação de Akaike e de Informação Bayesiano de Schwarz. O modelo com 18 classes heterogêneas foi o que melhor se ajustou às variâncias residuais, de acordo com os testes estatísticos, porém, o modelo com função de variância de quinta ordem também mostrou-se apropriado. Os valores de herdabilidade direta estimados foram maiores que os encontrados na literatura, variando de 0,04 a 0,53, mas seguiram a mesma tendência dos estimados pelas análises unicaracterísticas. A seleção para peso em qualquer idade melhoraria o peso em todas as idades no intervalo estudado.

Palavras-chave: classes de variâncias residuais, função de co-variância, polinômios ortogonais

\section{Estimates of covariance components for body weights from birth to 365 days of age in Guzera cattle, using random regression models}

\begin{abstract}
A total of 19,770 body weight records of Guzera cattle, measured from birth to 365 days of age and supplied by the Brazilian Zebu Breeders Association, was analyzed with the following objectives of: 1) to compare different residual variances through step functions with 1, 18, 28 and 53 classes and through variance functions with orders ranging from two to five using ordinary polynomials and 2) to estimate covariance functions considering different orders for direct additive genetic effects, animal permanent environmental and maternal permanent environmental effects, using random regression models. The random effects included were modeled by regression on Legendre Polynomials with orders ranging from linear to quartic. The models were compared through the likelihood ratio test, Akaike's information criterion and the Schwarz's Bayesian information criterion. The model with 18 heterogeneous classes was the one that best fitted the residual variances, according to the statistical tests; however, the model with variance function of $5^{\text {th }}$ order also showed to be appropriate. The direct heritability estimates were higher than those found in literature, ranging from 0.04 to 0.53 , showing similar trends when compared to those estimated using univariate models. Selection on body weight in any age should improve the body weight in all ages in the studied interval.
\end{abstract}

Key Words: covariance function, orthogonal polynomials, residual variance classes

\section{Introdução}

Os modelos de regressão aleatória e as funções de co-variâncias têm despertado interesse de pesquisadores da área de melhoramento genético animal em análises de dados que podem ser tomados repetidamente ao longo da vida do animal, como o peso corporal (Meyer, 1998a, 1999, 2000, 2001, 2002; Albuquerque \& Meyer, 2001; Albuquerque, 2003; Sakaguti et al., 2002; Cyrillo et al., 2004). Essa metodologia permite o ajuste de curvas de crescimento aleatórias para cada animal, expressas como desvios de uma curva média da população ou de grupos de indivíduos

Este artigo foi recebido em 5/7/2007 e aprovado em 17/6/2008. Correspondências devem ser enviadas para: saquei@fcav.unesp.br 
(Schaeffer, 1996). Uma vez que os efeitos aleatórios são descritos por funções contínuas nos modelos de regressão aleatória, Meyer \& Hill (1997) e Meyer (1998), demonstraram a equivalência entre funções de co-variância e os modelos de regressão aleatória. Assim, as funções de co-variâncias podem ser, diretamente, estimadas por análises de máxima verossimilhança restrita ao se aplicarem modelos de regressão aleatória em dados de dimensão infinita (Meyer, 1998a).

Os primeiros estudos utilizando modelos de regressão aleatória (Jamrozik \& Schaeffer, 1997; Jamrozik et al., 1997) desconsideravam estrutura heterogênea de variâncias residuais, o que proporcionava superestimações das variâncias genéticas aditivas. A modelagem considerando heterogeneidade de variâncias residuais para cada idade, no entanto, pode melhorar a partição da variação total, mas também proporciona aumento no número de parâmetros a serem estimados no processo de aumento da função de verossimilhança (El Faro \& Albuquerque, 2003).

$\mathrm{Na}$ busca por modelos de regressão aleatória mais parcimoniosos, alternativas têm sido sugeridas e uma delas é a utilização de estruturas de variâncias residuais diferentes, pelo agrupamento de classes com variações residuais semelhantes ou por funções de variâncias residuais, utilizando polinômios ordinários ou ortogonais (Albuquerque \& Meyer, 2001; El Faro \& Albuquerque, 2003).

Os objetivos neste trabalho foram: a) comparar diferentes estruturas de variâncias residuais em modelos de regressão aleatória visando reduzir o número de parâmetros a serem estimados, o que facilita o processo de convergência e a obtenção dos componentes de variâncias, e b) estimar funções de co-variância de diferentes ordens para os efeitos genético direto, genético materno e de ambiente permanente de animal e de mãe, bem como parâmetros genéticos para os pesos corporais usando modelos de regressão aleatória.

\section{Material e Métodos}

Utilizaram-se dados de 19.770 pesos corporais, medidos entre o nascimento e os 365 dias de idade em 4.920 bovinos Guzerá nascidos no período de 1979 a 1999, provenientes de 1.759 grupos de contemporâneos, filhos de 466 touros e 3.048 vacas, pertencentes ao banco de dados da Associação Brasileira dos Criadores de Zebu.

Análises unicaracterísticas foram realizadas considerando arquivos de dados com animais pesados ao nascimento e em idades de 1 a 60, 30 a 90, 60 a 120,.., 300 a 360 e 330 a 390 dias. As idades, em cada arquivo analisado, se sobrepõem para que existam ligação e correlação entre elas, tornando a curva mais abrangente. Os efeitos fixos considerados foram grupo de contemporâneos (formado por proprietário, fazenda do proprietário, criador, fazenda do criador, sexo, ano e mês do nascimento, regime alimentar, condição de criação, categoria e ano e mês da pesagem) e as co-variáveis foram idade da vaca ao parto (linear e quadrática) e idade do animal à pesagem (linear). Os efeitos aleatórios estudados foram o genético direto, o genético materno e o de ambiente permanente materno.

As estimativas dos componentes de variância foram obtidas pela máxima verossimilhança restrita, utilizando-se o programa computacional MTDFREML, descrito por Boldman et al. (1993).

Nas análises empregando modelos de regressão aleatória, os pesos corporais foram agrupados em classes de 7 dias de idade (para aumentar o número de observações em cada idade), resultando em 53 idades diferentes com mínimo de três e máximo de seis pesagens por animal. Nesses modelos, os efeitos aleatórios, genético direto, genético materno e de ambiente permanente de animal e de mãe foram modelados por regressões polinomiais em escala de Legendre, com ordens variando de linear a quártica. Apesar de o arquivo de dados analisado apresentar até seis registros por animal, havia pequeno número de informações com seis pesagens (registros), o que impossibilitou empregar polinômio de quinta ordem.

Os efeitos fixos incluídos nos modelos de regressão aleatória foram os mesmos considerados nas análises unicaracterísticas, com exceção da idade do animal à pesagem, que foi modelada utilizando-se regressão cúbica sobre polinômios ortogonais da idade (Legendre).

O polinômio ortogonal de ordem cúbica para a regressão fixa foi escolhido, pois o quadrado médio do resíduo para esta ordem foi menor em comparação às ordens linear e quadrática e pouco diferiu da ordem quártica.

Diversas estruturas de variâncias residuais foram tes tadas considerando-se: homogeneidade de variância residual (1 classe); heterogeneidade de variâncias residuais por: a) variâncias residuais agrupadas e modeladas com 18 , 28 e 53 classes de idade. O agrupamento das classes foi realizado com base no modelo com 53 diferentes classes, em que as idades com estimativas de variâncias residuais próximas foram agrupadas na mesma classe; b) funções de variância modeladas por polinômios ordinários: ordens quadrática; cúbica; quártica e quíntica.

No estudo das estruturas de variâncias residuais, foi considerada ordem cúbica para a função de regressão para os efeitos genético direto, de ambiente permanente de animal e de mãe. Quando utilizadas funções de variância, as 
variâncias residuais foram estimadas pelos coeficientes de regressão das funções de variância, representados por:

$\sigma_{e_{i}}^{2}=\sigma_{e_{o}}^{2}\left(1+\sum_{r=1}^{q} \beta_{r} t_{i j}^{r}\right)$

em que $\sigma_{\text {eo }}^{2}=$ variância do intercepto; $\beta_{\mathrm{r}}=\mathrm{r}$ coeficientes de regressão das funções de variância de ordem q; e $\mathrm{t}_{\mathrm{ij}}=$ idades $\mathrm{i}$ para cada animal $\mathrm{j}$.

O modelo geral de regressão aleatória é representado por:

$Y_{i j}=F+\sum_{m=0}^{k_{j}-1} \beta_{m} \phi_{m}\left(t_{i}\right)+\sum_{m=0}^{k_{a}-1} \alpha_{j m} \phi_{m}\left(t_{i j}\right)+\sum_{m=0}^{k_{m}-1} \mu_{j m} \phi_{m}\left(t_{i j}\right)+\sum_{m=0}^{k_{a n}-1} \gamma_{j m} \phi_{m}\left(t_{i j}\right)+\sum_{m=0}^{k_{k m}-1} \lambda_{j m} \phi_{m}\left(t_{i j}\right)+\varepsilon_{i j}$

em que: $\mathrm{Y}_{\mathrm{ij}}=$ peso corporal na i-ésima idade, pertencente ao j-ésimo animal; $F=$ conjunto de efeitos fixos; $\beta_{\mathrm{m}}=$ conjunto de $m$ regressores fixos para a curva média da população; $\phi_{m}\left(t_{i}\right)=$ função de regressão de ordem $m$, que descreve a curva média da população, de acordo com a idade (i); $\phi_{\mathrm{m}}\left(\mathrm{t}_{\mathrm{ij}}\right)=$ funções de regressão que descrevem a trajetória de cada indivíduo j, de acordo com a idade (i), para os efeitos genético direto, genético materno, de ambiente permanente de animal e de mãe; $\alpha_{j m}, \mu_{j m}, \gamma_{j m}, \lambda_{j m}=$ regressores aleató rios genético direto, genético materno e de ambiente permanente de animal e mãe, para cada animal $\mathrm{j} ; \mathrm{k}_{\mathrm{b}}=$ ordem do polinômio utilizado na regressão fixa (4); $\mathrm{k}_{\mathrm{a}}, \mathrm{k}_{\mathrm{m}}, \mathrm{k}_{\mathrm{apa}}$, $\mathrm{k}_{\mathrm{apm}}=$ ordens dos polinômios utilizados para os efeitos aleatórios, sendo $\mathrm{k}_{\mathrm{a}}$ e $\mathrm{k}_{\mathrm{apa}}=4$ e $5 ; \mathrm{k}_{\mathrm{apm}}=2,3$ e $4 ; \mathrm{k}_{\mathrm{m}}=0$ e 2; $\varepsilon_{\mathrm{ij}}=$ erro aleatório associado a cada idade i do animal j.

A partir das matrizes de coeficientes de regressão (K), foram obtidas as matrizes de autovalores e autovetores, $\mathrm{K}=\mathrm{EDE}^{\prime}$, em que E é uma matriz com $\mathrm{k}$ autovetores e D, uma matriz diagonal com $\mathrm{k}$ autovalores e, a cada autovalor, existe um autovetor relacionado. Cada autovalor é responsável por uma proporção da variação total existente e, quanto maior essa proporção, maior a variação genética relacionada ao autovalor. Parâmetros muito correlacionados entre si apresentam autovalores próximos de zero. Em comparação às multicaracterísticas, as variáveis, em medidas repetidas, podem ser ordenadas ao longo de uma trajetória e, neste caso, a transformação das variáveis descritas para cada autovetor pode ser escrita como uma função contínua da idade e isto é indicado como autofunção (Kirkpatrick \& Heckman, 1989). As autofunções são obtidas pelo produto entre a matriz de coeficientes do polinômio ortogonal (f), de ordem $\mathrm{t} x \mathrm{k}$, e a matriz com os autovetores (E), de ordem $\mathrm{kx}$ $\mathrm{k}$, em que t é o número de idades diferentes do animal e k é a ordem da função de co-variâncias (Van der Werf \& Schaeffer, 1997).

Os componentes de variância foram estimados pelo método da máxima verossimilhança restrita (REML) usando a opção DXMRR do pacote estatístico DFREML (Meyer,1998).
A comparação entre os modelos de regressão aleatória foi realizada pelo teste da razão de verossimilhança (LRT) e pelos critérios de Informação de Akaike (AIC) e de Informação Bayesiano de Schwarz (BIC), de acordo com Wolfinger (1993). Os testes AIC e BIC permitem a comparação entre modelos não-aninhados e penalizam modelos com maior número de parâmetros. No modelo Bayesiano de Schwarz, essa penalidade é mais rigorosa, o que favorece modelos mais parcimoniosos.

\section{Resultados e Discussão}

No estudo das estruturas de variâncias residuais, os parâmetros estimados a partir da regressão polinomial de Legendre foram $58,2128 \mathrm{~kg}$ para o intercepto, $124,5834 \mathrm{~kg}$ para o coeficiente de regressão linear, $-10,4656 \mathrm{~kg}$ para o coeficiente de regressão quadrática e $9,3399 \mathrm{~kg}$ para o coeficiente de regressão cúbica.

Comparando os modelos com classes de variâncias residuais, de acordo com LRT, verificou-se que o modelo considerando homogeneidade de variância residual foi inadequado e diferiu significativamente $(\mathrm{P}<0,01)$ dos demais (Tabela 1).

Os modelos com classes de variâncias heterogêneas $(18,28$ e 53) não diferiram significativamente $(\mathrm{P}>0,01)$ entre si com o aumento no valor da função de verossimilhança, porém, o critério de Informação Bayesiano de Schwarz considerou o modelo com 18 classes de variâncias residuais como mais adequado, enquanto o critério de Informação de Akaike considerou mais adequado o modelo com 28 classes de variâncias residuais. Entre os modelos estudados com funções de variâncias residuais, o melhor, de acordo com os três critérios empregados (LRT, AIC e BIC), foi o modelo com polinômio ordinário de quinta ordem.

As estimativas de desvio-padrão fenotípico, de ambiente permanente de animal e de mãe apresentaram tendência de crescimento do nascimento aos 365 dias de idade, enquanto, para o efeito genético aditivo direto, verificou-se aumento do nascimento aos 266 dias de idade, com decréscimo a partir deste ponto até 365 dias de idade (Figura 1).

Observou-se aumento desproporcional dos desviospadrão fenotípico e de ambiente permanente de animal no final da curva de crescimento, provavelmente relacionado à ordem de ajuste aplicada para modelar o efeito de ambiente permanente de animal, indicando que ordens maiores deverão ser testadas. Polinômios de ordens mais altas são necessários para modelar as mudanças nas variâncias de ambiente permanente (Brotherstone et al., 2000; Albuquerque, 2003).

Os desvios-padrão residuais foram maiores no início e decresceram a partir de 190 dias de idade, com exceção 
Tabela 1 - Número de parâmetros (p), valor da função de máxima verossimilhança (Log L), teste de razão de verossimilhança (LRT) e Critérios de Informação de Akaike (AIC) e de Informação Bayesiano de Schwarz (BIC) para os modelos utilizando homogeneidade e heterogeneidade de variância residual (classes: 18, 28 e 53; funções de variâncias: quadrática a quíntica)

\begin{tabular}{|c|c|c|c|c|c|}
\hline Modelo & $\mathrm{p}$ & $\log \mathrm{L}$ & $\mathrm{AIC}$ & $\mathrm{BIC}$ & LRT \\
\hline & & & de de variân & & \\
\hline \multirow[t]{2}{*}{ (1) 1} & 31 & $-53779,69$ & 107621,39 & 107866,04 & - \\
\hline & \multicolumn{4}{|c|}{ Heterogeneidade de variâncias residuais } & \\
\hline \multicolumn{6}{|l|}{ Classes } \\
\hline (2) 18 & 48 & $-52911,46$ & 105918,92 & 106297,74 & $(2-1) 868,23 *$ \\
\hline (3) 28 & 58 & $-52900,80$ & 105917,60 & 106375,34 & (3-2) 10,65 NS \\
\hline \multirow[t]{2}{*}{ (4) 53} & 83 & $-52875,80$ & 105917,61 & 106572,56 & $(4-3) 25,03 \mathrm{NS}$ \\
\hline & \multicolumn{4}{|c|}{ Funções de variâncias residuais } & \\
\hline (1) Quadrática & 33 & $-53234,39$ & 106534,78 & 106795,22 & - \\
\hline (2) Cúbica & 34 & $-53071,99$ & 106211,99 & 106480,13 & $(2-1) 162,39 *$ \\
\hline (3) Quártica & 35 & $-53056,98$ & 106183,96 & 106460,18 & $(3-2) \quad 15,01 *$ \\
\hline (4) Quíntica & 36 & $-53041,75$ & 106155,51 & 106439,62 & $(4-3) \quad 15,23 *$ \\
\hline
\end{tabular}

* = significativo $(\mathrm{P}<0,01) ;{ }^{\mathrm{NS}}=$ não-significativo.

do modelo com função de variância residual quadrática, que apresentou decréscimo nos valores do nascimento aos 365 dias de idade. Os desvios-padrão do efeito genético aditivo direto apresentaram valores maiores que os do efeito de ambiente permanente de animal no intervalo entre 119 e 294 dias de idade. A maior diferença foi observada para o modelo com função de variância de ordem quártica (Figura 2).

Olori et al. (1999), estudando diversas estruturas de variância residual em curva de lactação, observaram que mudanças na forma de modelar as variâncias residuais praticamente não afetaram as estimativas de variâncias para o efeito genético direto. No entanto, verificou-se neste trabalho que as estimativas de desvio-padrão obtidas para os efeitos genético aditivo direto e de ambiente permanente de animal foram, suavemente, influenciadas pela forma de modelar a estrutura de variância residual quando os três melhores modelos ( 18 e 28 classes e função de variância de quinta ordem) foram comparados. Esta mesma tendência foi observada por Albuquerque (2003) em estudo com pesos corporais de bovinos Nelore.

Comparando o modelo com homogeneidade de variância residual aos modelos com heterogeneidade residual (classes e funções de variância), verificaram-se valores de desviopadrão genético direto maiores para o modelo com homogeneidade residual, como observado por Jamrozik \& Schaeffer (1997) nos primeiros estudos com regressão aleatória em que desconsideravam heterogeneidade de variância residual.

Os modelos com 18, 28 e 53 classes de variâncias residuais apresentaram estimativas de desvios-padrão fenotípico, aditivo direto e de ambiente permanente de animal e de mãe praticamente idênticas, sugerindo que o modelo com 18 classes poderia ser escolhido como o mais adequado, juntamente com o modelo com função de variância de quinta ordem.

Com a redução do número de parâmetros estimados, os modelos se tornam parcimoniosos, proporcionando economia do tempo necessário para a convergência das análises.

De acordo com os resultados obtidos, não há necessidade de considerar todas as 53 variâncias residuais. É possível modelar as variâncias com 18 classes ou função de variância de quinta ordem, proporcionando assim ajustes parcimoniosos, principalmente se as ordens das funções de co-variância para os efeitos genético direto e de ambiente permanente forem maiores.

Funções de co-variância de diferentes ordens para os efeitos genético direto, genético materno e de ambiente permanente de animal e de mãe também foram estudadas.

Independentemente da modelagem realizada no resíduo, a inclusão do efeito genético materno (ordem linear) não aumentou significativamente o valor da função de verossimilhança (Tabela 2). Segundo Meyer (1992), quando apenas um dos efeitos maternos é considerado no modelo de análise, a maior parte da variação materna é, provavelmente, considerada. É provável também que a estrutura do conjunto de dados estudado não permitiu a correta separação e estimação dos efeitos maternos. Problema semelhante foi observado por Pelicioni et al. (2003) com o mesmo conjunto de dados, em análises unicaracterísticas. Esses autores verificaram que, no modelo contendo os dois efeitos maternos (genético e de ambiente permanente), quando um deles era medido, o outro se igualava a zero, ocorrendo troca de posições ao longo das idades, o que dificulta a separação e estimação destes efeitos. 
Homogeneidade de variância residual

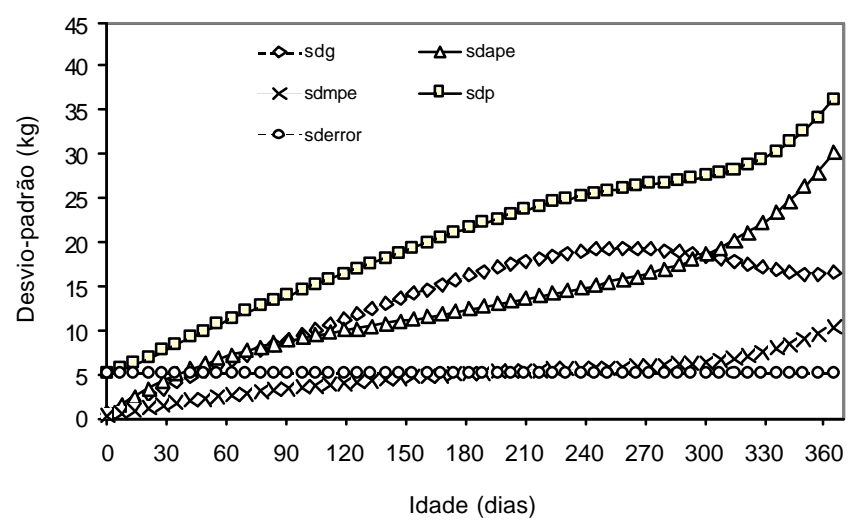

28 classes de variância residual

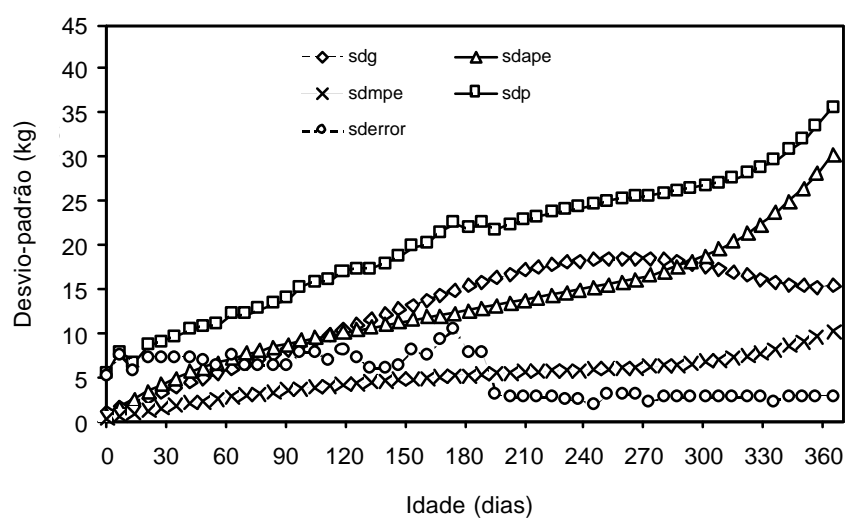

18 classes de variância residual

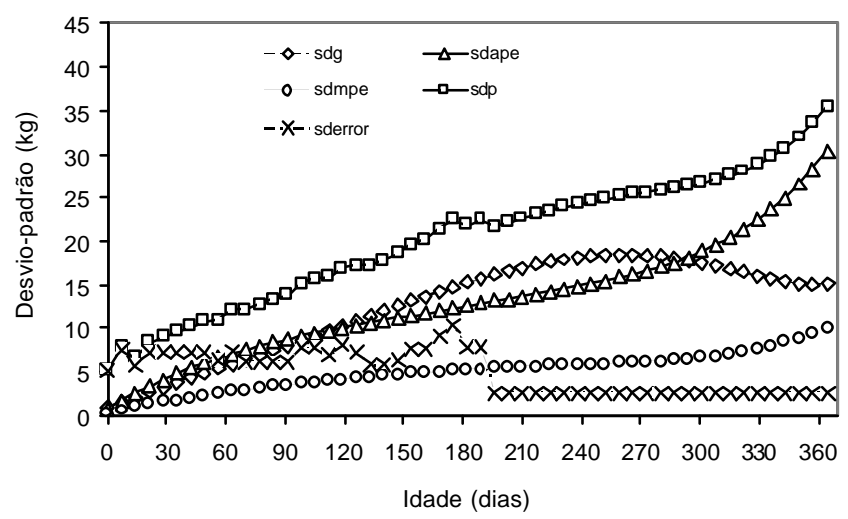

53 classes de variância residual

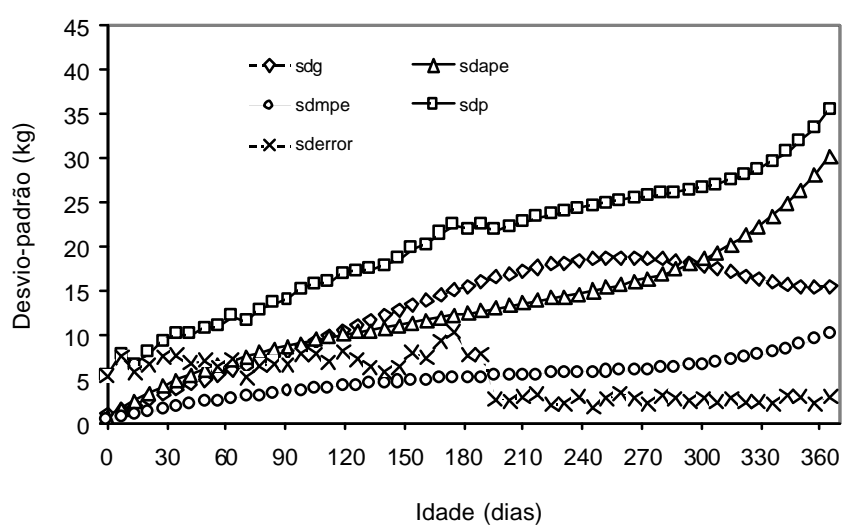

Figura 1 - Estimativas de desvios-padrão fenotípico (sdp), genético direto (sdg), ambiente permanente de animal (sdape), ambiente permanente de mãe (sdmpe) e residuais (sderror) para os modelos com homogeneidade e heterogeneidade de variâncias residuais (18, 28 e 53 classes).

De acordo com LRT, entre os modelos aninhados, houve diferença significativa $(\mathrm{P}<0,01)$ quando as ordens 4 e 5 para os efeitos genético direto e de ambiente permanen te de animal foram comparadas. Entre os modelos com ordem 5 para os efeitos genético direto e de ambiente permanente de animal, e diferentes ordens para os efeitos genético materno (zero e 2) e de ambiente permanente materno (2, 3 e 4), não houve diferença significativa pelo teste LRT (Tabela 2).

Para os testes AIC e BIC, quanto menor o valor obtido, melhor a qualidade do ajuste proporcionada pelo modelo.

Neste estudo, o modelo AIC considerou os modelos 5052_18_CVR (ordens de ajuste 5 para o efeito genético direto, zero para o genético materno, 5 para o de ambiente permanente de animal e 2 para o de ambiente permanente materno, com 18 classes de variâncias residuais) e 5052_28_CVR (ordens de ajuste 5 para o efeito genético direto, zero para o genético materno, 5 para o de ambiente permanente de animal e 2 para o de ambiente permanente materno, com 28 classes de variâncias residuais) como os melhores. No entanto, no modelo BIC, que atribui maior penalidade aos modelos mais parametrizados, o modelo mais adequado foi o 5052_FV5 (ordens de ajuste 5 para o efeito genético direto, zero para o genético materno, 5 para o de ambiente permanente de animal e 2 para o de ambiente permanente materno, com função de variância residual de quinta ordem (Tabela 2).

Observa-se discordância entre os teste AIC e BIC, porém as ordens 5052 das funções de co-variância para os efeitos estudados não diferiram.

A redução nas ordens dos polinômios realizada, apenas para o efeito de ambiente permanente materno, pode ser explicada, uma vez que as ordens mais elevadas para este efeito ( 3 e 4 ) apresentaram autovalores com pequena variação, próximos de zero, sugerindo a redução da ordem, o que possibilitou a obtenção de modelos mais parcimoniosos.

Os desvios-padrão fenotípicos estimados pelos modelos de regressão aleatória (Figura 3 ) foram próximos aos obtidos pelas análises unicaracterística, porém o final da variação apresentou aumento acentuado das estimativas. 


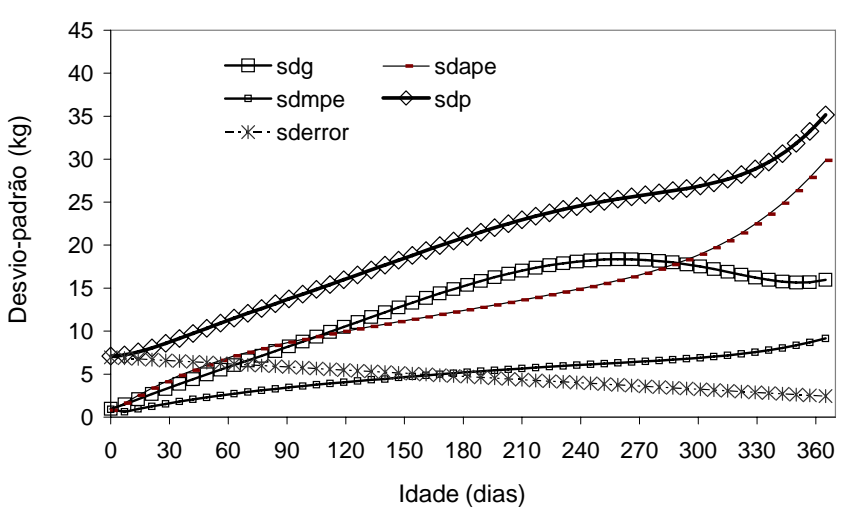

Quártica

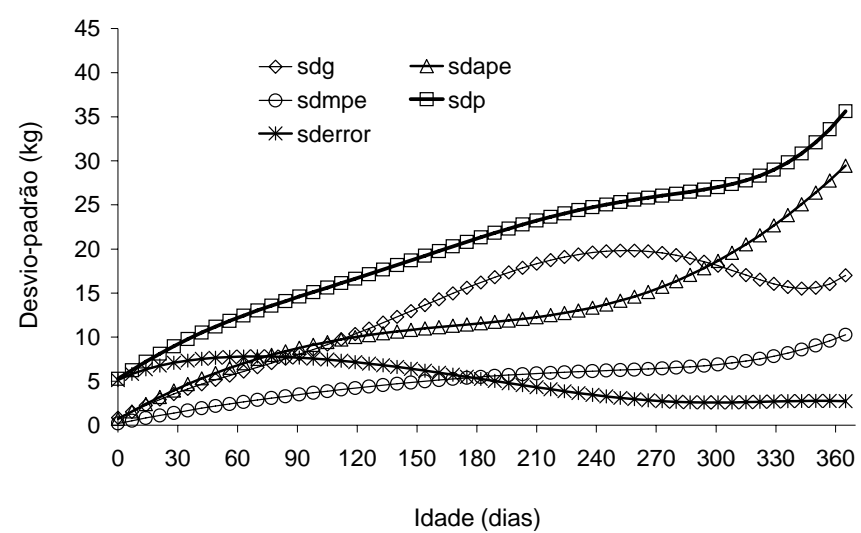

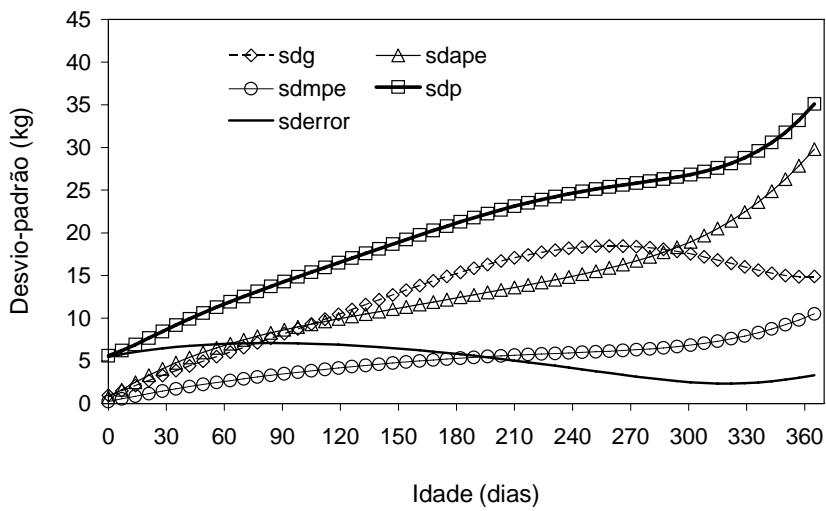

Quíntica

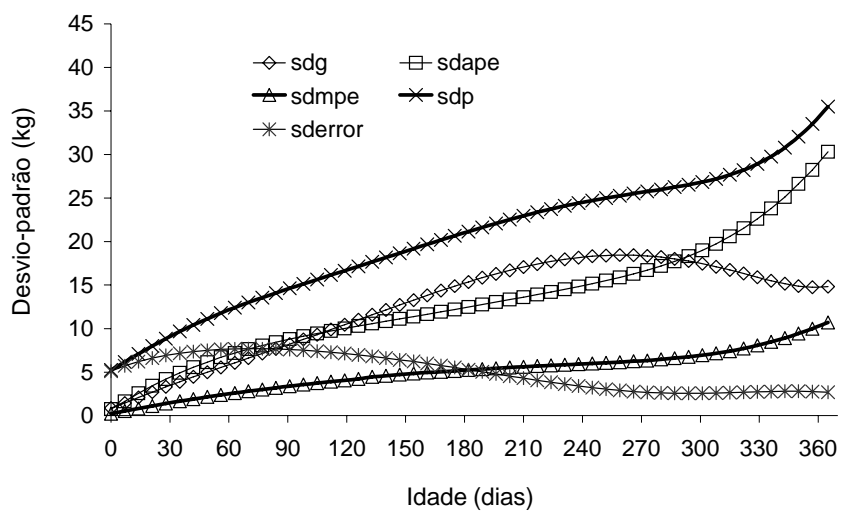

Figura 2 - Estimativas de desvios-padrão fenotípico (sdp), genético direto (sdg), de ambiente permanente de animal (sdape), de ambiente permanente de mãe (sdmpe) e residuais (sderror) para os modelos com funções de variâncias residuais de ordens quadrática a quíntica.

Tabela 2 - Número de parâmetros (p), valor da função de máxima verossimilhança (Log L), teste de razão de verossimilhança (LRT), critérios de Informação de Akaike (AIC) e de Informação Bayesiano de Schwarz (BIC) para os modelos utilizando diferentes ordens de ajuste para os efeitos genéticos direto e materno, de ambiente permanente de animal e de mãe

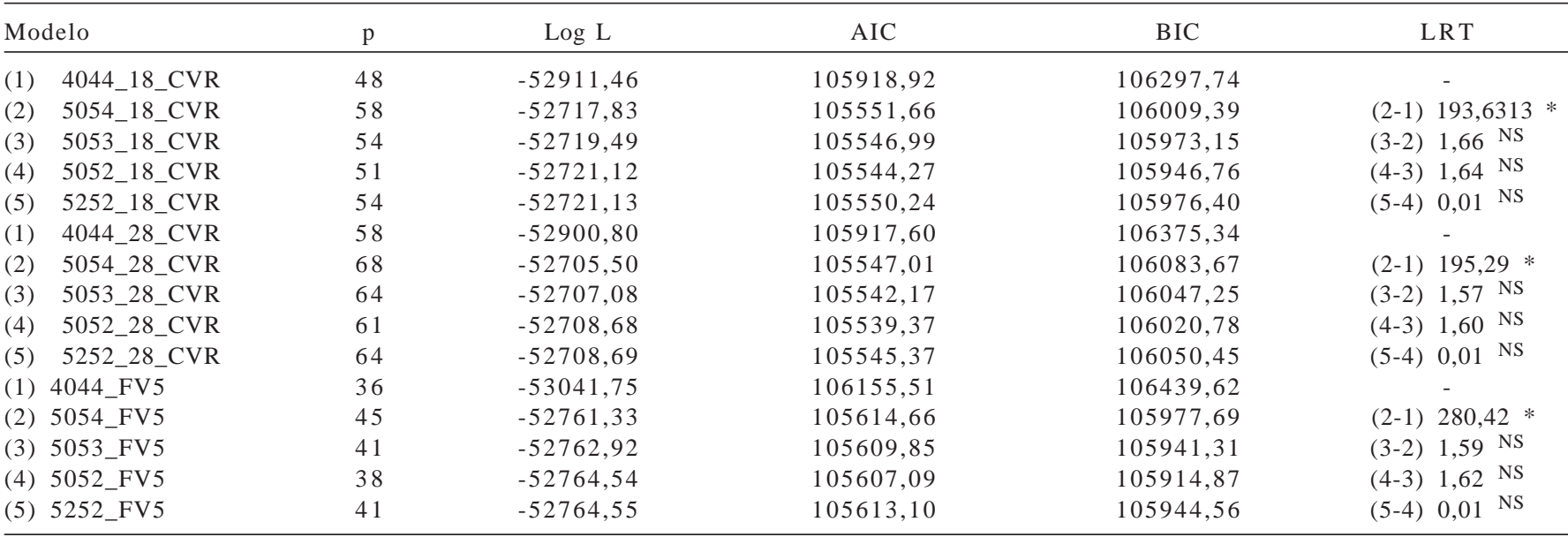

CVR = classes de variâncias residuais; FV5 = funções de variância de quinta ordem; $5252 \_18 \_$CVR = ordens de ajuste 5 para o efeito genético direto, 2 para o genético materno, 5 para o de ambiente permanente de animal e 2 para o ambiente permanente materno, respectivamente, com 18 classes de variâncias residuais; $*$ = significativo $\mathrm{P}<0,01,{ }^{N S}=$ não-significativo 

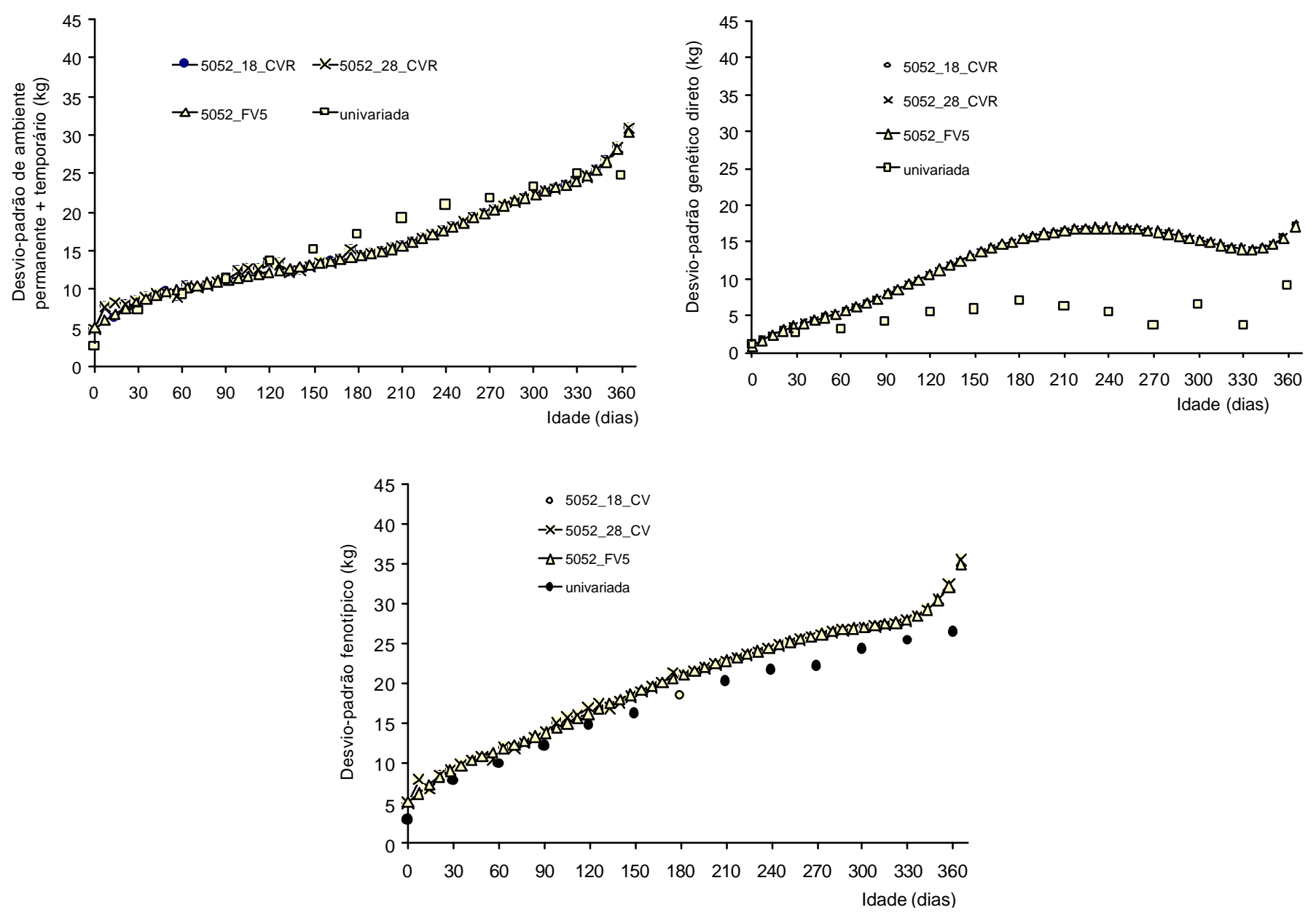

Figura 3 - Estimativas de desvios-padrão fenotípico, genético direto e de ambientes permanente + temporário estimados para o modelo 5052 (ordens de ajuste 5 para o efeito genético direto, 0 para o genético materno, 5 para o de ambiente permanente de animal e 2 para o de ambiente permanente materno) com 18 e 28 classes de variâncias residuais (CVR) e função de variância de quinta ordem (FV5) e para as análises unicaracterísticas.

De acordo com Meyer (1998a), as diferenças nas variâncias fenotípicas entre modelos de regressão aleatória e unicaracterística, principalmente nas idades finais, podem ser reflexo, ao menos em parte, de vieses na amostra, como conseqüência do descarte nas fases pós-desmama. Outra hipótese seria a necessidade de ordens mais elevadas para os efeitos genético direto e de ambiente permanente de animal.

As estimativas de desvio-padrão genético direto apresentaram valores mais altos para os modelos de regressão aleatória, a partir de 90 dias de idade, em comparação às análises unicaracterísticas.

Para os desvios-padrão dos ambientes permanente + temporário, verificou-se comportamento semelhante das estimativas para os dois métodos estudados, do nascimento aos 120 dias e de 270 a 330 dias de idade. Provavelmente, nas análises unicaracterísticas, o efeito genético direto foi con fundido com os efeitos de ambiente (permanente e temporário), proporcionando estimativas de desvio-padrão menores para o efeito genético direto no período entre 120 e 270 dias de idade.

Foi possível verificar também que os modelos com $18 \mathrm{e}$ 28 classes de variâncias residuais e função de variância de quinta ordem apresentaram estimativas semelhantes para todos os efeitos estudados (Figura 4), sugerindo que qualquer um desses modelos poderia ser empregado. Entretanto, se houver interesse na redução do número de parâmetros estimados e do tempo necessário para a realização das análises, os modelos com 18 classes e função de variância de quinta ordem poderiam ser adotados.

As estimativas de herdabilidade direta (Figura 5), de 0,03 a 0,53 , foram muito semelhantes para os três modelos estudados, empregando regressão aleatória. De modo geral, seguiram a mesma tendência das análises unicaracterística, no entanto, o início e o final da curva apresentaram padrões diferentes. 

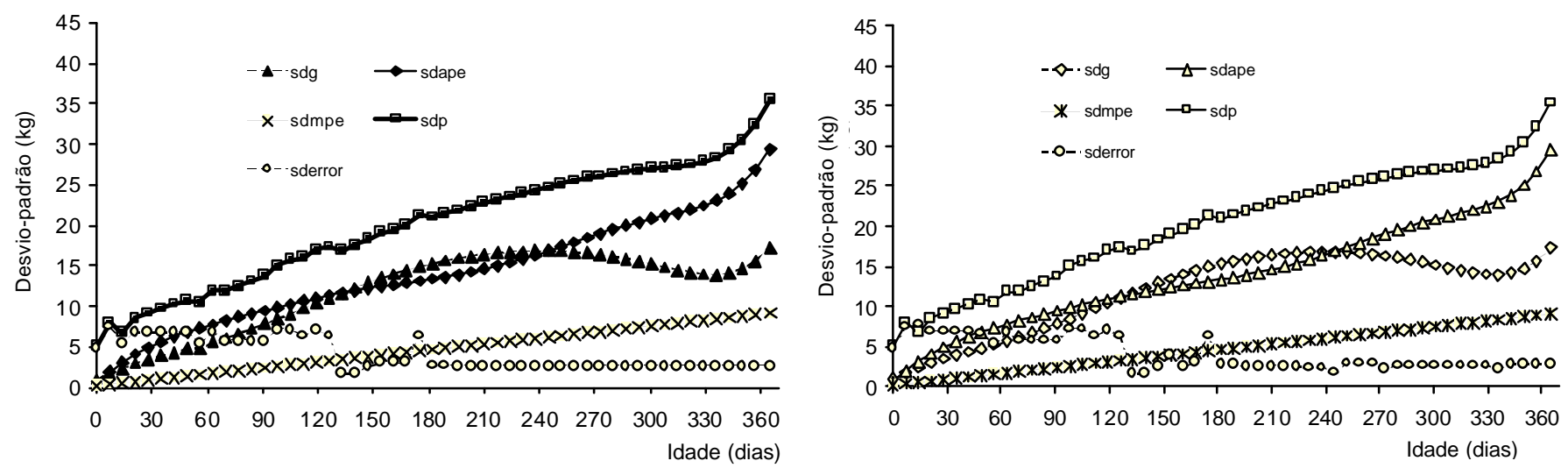

5052_FV5

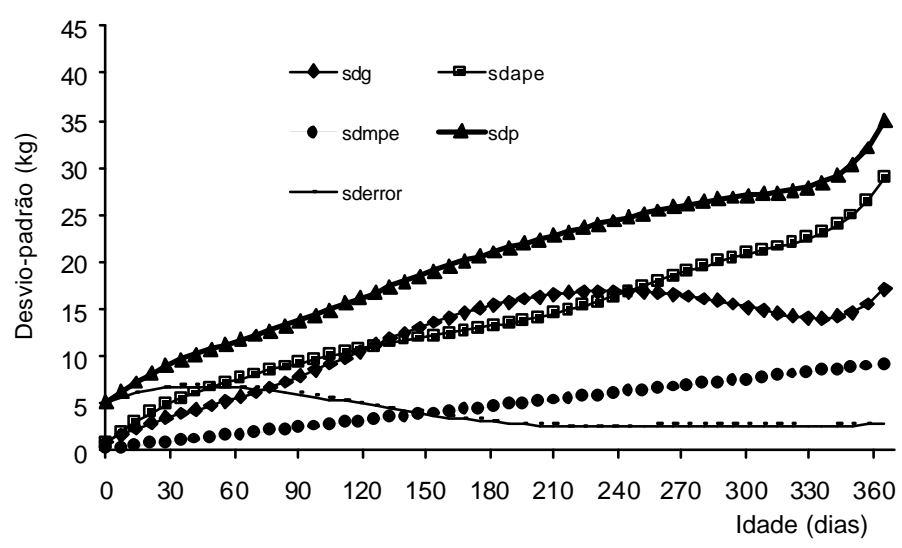

Figura 4 - Estimativas de desvios-padrão genético aditivo direto (sdg), de ambiente permanente de animal (sdape), de ambiente permanente de mãe (sdmpe), fenotípico (sdp) e residual (sderror) da curva de crescimento de bovinos Guzerá para os modelos 5052 (ordens de ajuste 5 para o efeito genético direto, 0 para o genético materno, 5 para o de ambiente permanente de animal e 2 para o de ambiente permanente materno) com 18 e 28 classes de variâncias residuais (CVR) e função de variância de quinta ordem (FV5).

Houve grande aumento nas estimativas de herdabilidade do nascimento $(0,03)$ aos 189 dias de idade $(0,53)$, que decresceram, a partir deste ponto, até 365 dias $(0,24)$. Essas estimativas foram mais altas que as descritas na literatura por Mercadante et al. (1995), no entanto, apresentaram a mesma tendência de comportamento das estimativas de herdabilidade obtidas pelas análises unicaracterísticas, embora com valo res sempre maiores que aqueles das unicaracterísticas, com exceção do peso ao nascer.

De acordo com Meyer (1992), modelos que não explicam o efeito genético materno podem produzir estimativas de variância genética direta mais altas e, como resultado, estimativas de herdabilidade direta também mais altas.

Resultados semelhantes foram relatados por Cyrillo et al. (2004), que trabalhando com pesos de machos Nelore e utilizando modelos de regressão aleatória e unicaracterística, encontraram estimativas de herdabilidade maiores para os modelos de regressão aleatória em comparação às estimadas por modelos unicaracterísticos.

Diferentemente, Albuquerque \& Meyer (2001), utilizando regressões aleatórias para analisar pesos de bovinos Nelore do nascimento aos 630 dias de idade, encontraram estimativas de herdabilidade maiores ao nascimento e em idades posteriores a 270 dias.

As correlações genéticas, fenotípicas e de ambiente permanente de animal e materno foram praticamente idênticas nos três melhores modelos (5052_18_CVR, 5052_28_CVRe 5052_FV5), com valores positivos variando de 0,148 a 1,000 para as correlações genéticas diretas, 0,405 a 1,000 para ambiente permanente de animal, 1,000 para ambiente permanente materno e 0,069 a 1,000 para as correlações fenotípicas.

As estimativas de correlações genéticas entre os pesos nas diferentes idades foram de moderadas a altas e com 

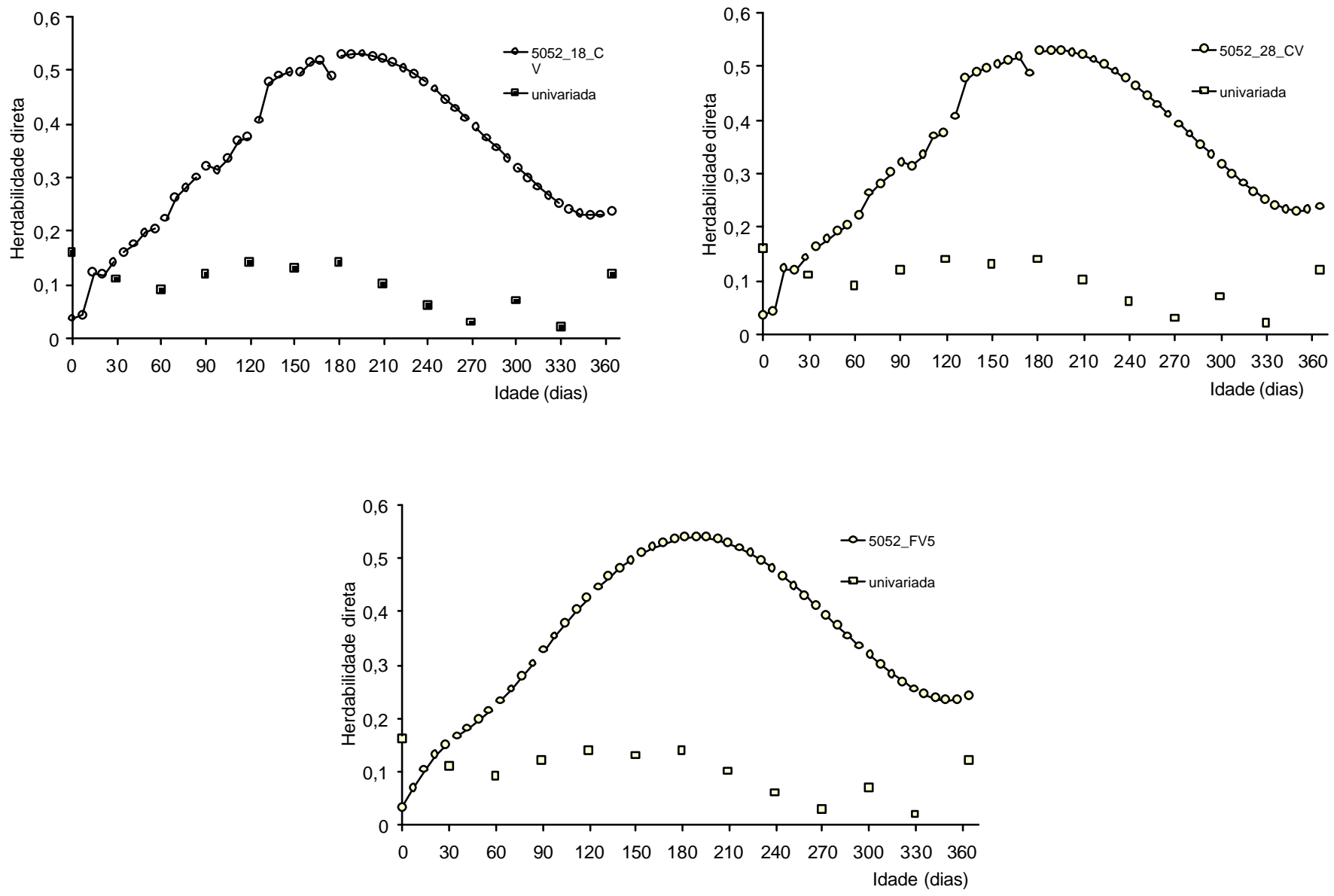

Figura 5 - Estimativas de herdabilidade direta para os pesos corporais de bovinos Guzerá, de acordo com os modelos de regressão aleatórias 5052 (ordens de ajuste 5 para o efeito genético direto, 0 para o genético materno, 5 para o de ambiente permanente de animal e 2 para o de ambiente permanente materno) com 18 e 28 classes de variâncias residuais (CVR) e função de variância de quinta ordem (FV5) e análises unicaracterísticas.

valores mais altos entre o peso ao nascer e os demais pesos nas idades iniciais e menores valores para os pesos em idades mais distantes. As estimativas de correlação fenotípica, no entanto, apresentaram tendência de crescimento com o aumento das idades. Entre o peso ao nascer e as demais idades, os valores foram baixos e variaram de 0,069 a 0,32.

As estimativas de correlação de ambiente permanente materno foram iguais a um em todas as idades, sugerindo que este efeito é o mesmo para os pesos nestas idades.

Os valores obtidos neste estudo confirmam os encontrados por Albuquerque \& Meyer (2001), que relataram correlações acima de 0,84 entre o peso ao nascer e as idades iniciais.

As estimativas de correlação de ambiente permanente de animal apresentaram valores mais altos nas idades mais próximas, com valores que reduziram à medida que as idades se distanciaram.

As correlações entre o intercepto e os coeficientes lineares para todos os efeitos foram positivas e altas
(Tabela 3), enquanto aquelas entre os interceptos e os coeficientes de regressão quadrática foram negativas, $-0,074$ para efeito de ambiente permanente de animal e de -0,750 para efeito genético direto.

Os autovalores para ambiente permanente de animal foram maiores que aqueles para o efeito genético direto. Para os dois efeitos, as maiores variações estiveram relacionadas ao primeiro, segundo e terceiro autovalores (Figura 6).

Segundo Van der Werf \& Schaeffer (1997), o sinal dos valores não é importante na interpretação das autofunções, mas sim a trajetória que esses valores assumem durante o crescimento dos animais, pois indica prováveis direções de seleção e a magnitude do autovalor associado a cada autofunção correspondente.

Os três maiores autovalores da matriz de coeficientes para o efeito genético aditivo foram responsáveis por $98,29 \%$ da variação genética total e o primeiro autovalor foi responsável por $87,93 \%$ da variação genética total. 
Tabela 3 - Estimativas de variâncias (diagonal), co-variâncias (abaixo da diagonal) e correlações (acima da diagonal) entre os coeficientes de regressão aleatória da matriz de coeficientes e seus autovalores $(\lambda)$ para os efeitos genético aditivo, de ambiente permanente do animal e de ambiente permanente da mãe

\begin{tabular}{|c|c|c|c|c|c|c|c|}
\hline & & 0 & 1 & 2 & 3 & 4 & $\lambda$ \\
\hline Genético & 0 & 238,1600 & 0,852 & $-0,750$ & $-0,300$ & 0,314 & 292,2580 \\
\hline \multirow[t]{4}{*}{ Aditivo } & 1 & 87,5765 & 44,3662 & $-0,485$ & $-0,344$ & 0,292 & 21,1718 \\
\hline & 2 & $-66,6621$ & $-18,5971$ & 33,1902 & 0,556 & $-0,325$ & 13,2691 \\
\hline & 3 & $-16,1596$ & $-7,98977$ & 11,1678 & 12,1596 & 0,419 & 5,6292 \\
\hline & 4 & 10,2598 & 4,12119 & $-3,9638$ & 3,0919 & 4,4800 & 0,0270 \\
\hline \multirow[t]{5}{*}{ Ambiente permanente de animal } & 0 & 303,105 & 0,801 & $-0,074$ & $-0,01$ & $-0,224$ & 371,9090 \\
\hline & 1 & 136,852 & 96,2898 & 0,396 & $-0,093$ & $-0,337$ & 59,6540 \\
\hline & 2 & $-7,9132$ & 23,9055 & 37,7592 & 0,371 & $-0,108$ & 33,5238 \\
\hline & 3 & $-0,9067$ & $-4,5746$ & 111,4106 & 25,0594 & 0,737 & 6,1976 \\
\hline & 4 & $-11,7325$ & $-9,9477$ & $-1,9973$ & 11,1074 & 9,0716 & $0,3751 \mathrm{E}^{-6}$ \\
\hline \multirow[t]{2}{*}{ Ambiente permanente de mãe } & 0 & 43,1805 & 1,000 & 56,5594 & & & \\
\hline & 1 & 24,0356 & 13,3789 & $0,1255 \mathrm{E}^{-5}$ & & & \\
\hline
\end{tabular}

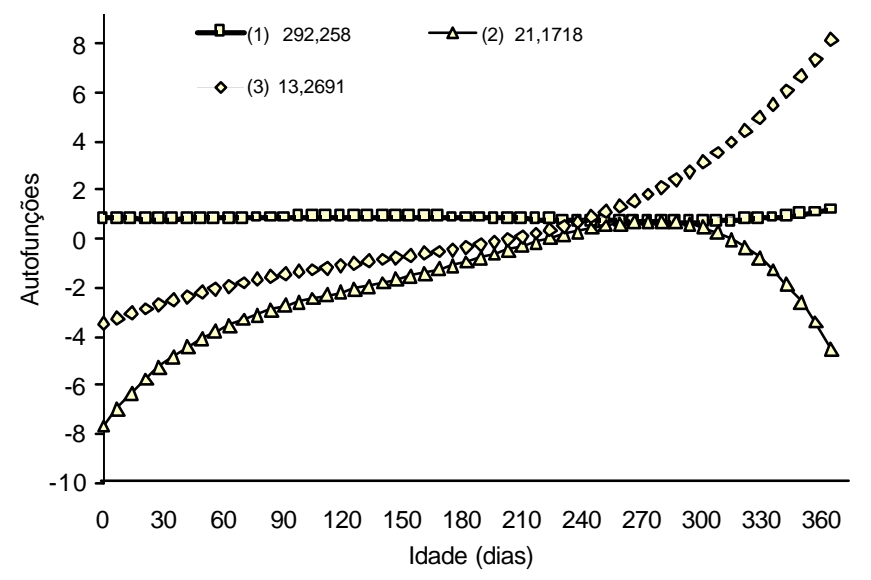

Figura 6 - Autofunções (1,2 e 3) estimadas para o efeito genético direto e seus respectivos autovalores $(292,258$; $21,1718$ e 13,2691$)$ para o modelo 5052_18_CVR (ordens de ajuste 5 para o efeito genético direto, 0 para o genético materno, 5 para o de ambiente permanente de animal e 2 para o de ambiente permanente materno, com 18 classes de variâncias residuais).

A primeira autofunção mostrou-se quase constante para todas as idades e indica que a maior parte da variação genética existente é explicada por um fator constante em todas as fases do crescimento e que a seleção baseada nesta autofunção aumentaria o peso em todas as idades, isto é, uma vez que seu autovalor é muito dominante, a seleção para peso em qualquer idade melhoraria o peso em todas as idades.

A segunda autofunção mostrou que a seleção sobre esta variável diminuiria o peso entre o nascimento e 217 dias e entre 315 e 365 dias de idade.

A terceira autofunção indica relação negativa e desfavorável entre os pesos até 203 dias e favorável a partir de 210 dias de idade. Provavelmente, a seleção sobre esta variável poderia ser usada para alterar a curva de cres- cimento, ou seja, selecionar para pesos menores no início e maiores no final da trajetória considerada.

Neste estudo o $2 \underline{0}$ e o $3 \underline{0}$ autovalores foram muito pequenos em relação ao 10 autovalor e sugerem que as possibilidades para alterar a curva de crescimento, com base em suas autofunções, poderiam ser limitadas.

\section{Conclusões}

A homogeneidade de variância residual não foi adequada para modelar o resíduo. Os modelos de regressão aleatória com ordens de ajuste 5 para o efeito genético direto, 0 para o genético materno, 5 para o de ambiente permanente de animal e 2 para o de ambiente permanente materno, com 18 classes de variâncias residuais e função de variância de quinta ordem foram mais adequados para descrever as mudanças nas variações nos pesos corporais ao longo das idades, proporcionando modelos parcimoniosos. O efeito genético materno não apresentou mudanças nas estimativas dos componentes de variância dos efeitos estudados. Os modelos de regressão aleatória proporcionaram maiores estimativas de herdabilidade direta em comparação às análises unicaracterísticas, com exceção do peso ao nascer. A autofunção estimada para o efeito genético aditivo sugere que a seleção praticada para o peso em qualquer idade traria progresso genético em todas as fases do crescimento.

\section{Literatura Citada}

ALBUQUeRQUe, L.G. Modelos de dimensão infinita, aplicados a características de crescimento de bovinos da raça Nelore. 2003. 83f. Tese (Livre Docência) - Faculdade de Ciências Agrárias e Veterinárias, Universidade Estadual Paulista, Jaboticabal, 2003. 
ALBUQUERQUE, L.G.; MEYER, K. Estimates of covariance functions for growth from birth to 630 days of age in Nelore cattle. Journal of Animal Science, v.79, p.2776-2789, 2001.

BOLDMAN, K.G.; KRIESE, L.A.; Van VLECK, L.D. et al. A set of programs to obtain estimates of variances and covariance. A manual for use of MTDFREML. Clay Center: USDA-ARS, 1993. $120 \mathrm{p}$

BROTHERSTONE, S.; WHITE, I.M.S.; MEYER, K. Genetic modelling of daily milk yield using orthogonal polynomials and parametric curves. Animal Science, v.70, p.416-417, 2000.

CYRILLO, J.N.S.G.; ALENCAR, M.M.; HAZOOK, A.G. et al. Modelagem e estimação de parâmetros genéticos e fenotípivos para pesos do nascimento à seleção (378 dias) de machos Nelore. Revista Brasileira de Zootecnia, v.33, n.6, p.1405-1415, 2004.

EL FARO, L.; ALBUQUERQUE, L.G. Utilização de modelos de regressão aleatória para produção de leite no dia do controle, com diferentes estruturas de variâncias residuais. Revista Brasileira de Zootecnia, v.32, n.5, p.1104-1113, 2003.

JAMROZIK, J.; SCHAEFFER, L.R. Estimates of genetic parameters for a test day model with random regression for yield traits of first lactation Holsteins. Journal of Dairy Science, v.80, p.762-770, 1997.

JAMROZIK, J.; KISTEMAKER, G.J.; DEKKERS, J.C.M. et al. Comparison of possible covariates for use in a random regression model for analyses of test day yields. Journal of Dairy Science, v.80, p.2550-2556, 1997.

KIRKPATRICK, M.; HECKMAN, N. A quantitative genetic model for growth, shape and other infinite-dimensional characters. Journal of Mathematical Biology, v.27, p.429-450, 1989.

MEYER, K. Variance components due to direct and maternal effects for growth traits of Australian beef cattle. Livestock Production Science, v.31, p.179-204, 1992.

MEYER, K.; HILL, W.G. Estimation of genetic and phenotypic covariance functions for longitudinal or "repeated" records by restricted maximum likelihood. Livestock Production Science, v.47, p.185-200, 1997.

MEYER, K. Estimating covariance functions for longitudinal data using a random regression model. Genetics, Selection and Evolution, v.30, p.221-240, 1998a.

MEYER, K. "DXMRR" - A program to estimate covariance functions for longitudinal data by REML. In: WORLD CONGRESS OF
GENETICS APPLIED TO LIVESTOCK PRODUCTION, 6., 1998, Armidale. Proceedings... Armidale, 1998.(CD-ROM).

MEYER, K. Estimates of genetic and phenotypic covariance functions for postweaning growth and mature weight of beef cows. Journal of Animal Breeding and Genetics, v.116, n.3, p.181-203, 1999.

MEYER, K. Random regression to model phenotypic variation in montly weights of Australian bef cows. Livestock Production Science, v.65, p.19-38, 2000.

MEYER, K. Estimates of direct and maternal covariance functions for growth of Australian beef calves from birth to weaning Genetics, Selection and Evolution, v.33, p.1-28, 2001

MEYER, K. Estimates of covariance functions for growth of Australian beef cattle from a large set of field data. In: WORLD CONGRESS ON GENETICS APPLIED TO LIVESTOCK PRODUCTION, 7., 2002, Montpellier. Proceedings... Montpellier, 2002. (CD-ROM).

OLORI, V.E.; HILL, W.G.; MCGUIRK, B.J. et al. Estimating variance components for test day milk records by restricted maximum likelihood with a random regression animal model. Livestock Production Science, v.61, p.53-63, 1999.

PELICIONI, L.C.; QUEIROZ, S.A.; ALBUQUERQUE, L.G. Estimativas de parâmetros genéticos para pesos ao nascer e mensais até 450 dias em bovinos Guzerá. Archivos Latinoamericanos de Producción Animal, v.11, n.1, p.34-39, 2003.

SAKAGUTI, E.S.; SILVA, M.A.; MARTINS, E.N. et al.Trajetória de crescimento e efeito da idade da vaca nos modelos de regressão aleatória de bovinos jovens da raça Tabapuã. Arquivos Brasileiros de Medicina Veterinária e Zootecnia, v.54, n.4, p.414-423, 2002.

SCHAEFFER, L.R. Tópicos avançados em melhoramento animal: random regression models. Jaboticabal: Universidade Estadual Paulista, 1996. p.25-33. (Notas do curso).

Van der WERF, J.H.J.; GODDARD, M.E.; MEYER, K. The use of covariance functions and random regressions for genetic evaluation of milk production based on test day records. Journal of Dairy Science, v.81, p.3300-3308, 1998.

WOLFINGER, R.D. Covariance structure in general mixed models. Communications in Statistics, v.22B, p.1079$1106,1993$. 